American Journal of Infectious Diseases 2 (3): 180-183, 2006

ISSN 1553-6203

(C) 2006 Science Publications

\title{
Nutritional Alterations in Drug Abusers With and Without HIV
}

\author{
Janet E. Forrester \\ Department of Public Health and Family Medicine, Tufts University School of Medicine \\ 136 Harrison Ave, Boston MA 02111
}

\begin{abstract}
Many studies have found that drug abusers have nutritional deficits, including weight deficits. The most plausible explanation for these deficits is dietary insufficiency. However, studies using objective measures of the dietary intake of drug abusers have failed to provide evidence of dietary insufficiency. Other mechanisms have rarely been examined. This article reviews the published literature on the nutritional status of drug abusers with and without HIV, with emphasis on dietary energy and macronutrient intake.
\end{abstract}

Key words: HIV, drug abuse, body mass index, nutrition, dietary intake

\section{INTRODUCTION}

The AIDS pandemic has led to an increased interest in the role of drug abuse on nutritional status and immunity. While it is recognized that drug abusers - both HIV positive and HIV-negative - frequently have nutritional deficiencies, it is not yet understood whether observed effects on nutrition and immunity are due directly to drugs of abuse per se or whether they are due to other, non-drug-related differences among groups.

Studies examining the nutritional status of drug abusers have shown variable results. Many, but not all studies have found drug abusers to have a lower average weight when compared to controls ${ }^{[1-11]}$. Factors that could account for discrepancies among studies include differences in the types and frequency of drugs abused, differences in rates of infections, such as HIV or hepatitis and differences between drug abusers who are hospitalized or enrolled in detoxification programs compared to community-living drug abusers. While dietary insufficiency is the most plausible cause of poor nutrition, studies using objective measures of dietary intake have failed to provide evidence that the diet of drug abusers can explain differences in nutritional status, including differences in weight ${ }^{[3,7,10,11]}$. Nondietary factors have rarely been examined ${ }^{[12]}$.

The effect of drug abuse on weight: A European study that predates the first reports of HIV found lower average weights among drug abusers compared to nondrug abusers ${ }^{[1]}$. Methadone maintained subjects had a lower body mass index (BMI) compared to controls. However, there was no difference in BMI between controls and heroin-maintained or heroin and methadone-maintained subjects. Needle exchange users in Glasgow, UK had a higher prevalence of low BMI $\left(<21 \mathrm{~kg} \mathrm{~m}^{-2}\right)$ compared to age-matched controls ${ }^{[6]}$.
Subjects were not tested for HIV, but the prevalence of HIV among drug abusers was reported to be low (1\%) at the time of the study. Hispanic, drug-abusing women in Connecticut, USA had a lower BMI than women who were not drug abusers ${ }^{[8]}$. The HIV status of the women was not reported.

A study conducted in Baltimore, USA, illustrates the importance of knowing the HIV status of drug abusers when examining the impact of drug abuse on nutrition $^{[7]}$. Involuntary weight loss of more than ten pounds in the previous six months was self-reported in $5 \%$ of HIV-negative drug abusers and $16 \%$ of HIVpositive drug abusers, suggesting that drug abuse compounds nutritional problems in persons infected with HIV. Working with HIV-infected persons in Boston, our own group found that women who were current intravenous drug users (IVDU) had a lower BMI when compared to women who had never used drugs. However, in the same study, no difference in BMI was found between HIV-positive IVDU men compared to HIV-positive men who had never used drug ${ }^{[10]}$. Accounting for differences in the stage of HIV disease did not alter the conclusions. Early stage HIV and infection with hepatitis did not account for differences in nutritional parameters between drug abusers and non-drug abusers in studies that collected information on infection status ${ }^{[2,12]}$. Thus, the impact of drug abuse may depend on the severity of concurrent disease.

It follows that malnutrition would be seen more often in hospitalized drug abusers than in communityliving drug abusers. Significant protein-energy malnutrition was found among drug abusers hospitalized for detoxification ${ }^{[2,5,9]}$ with even greater degrees of malnutrition among drug abusers who were admitted to hospital for other reasons, including infections ${ }^{[5]}$. Underlying infections related to drug abuse may contribute to malnutrition or malnutrition 
may increase susceptibility to infections. Studies of hospitalized drug abusers cannot distinguish between these two possibilities. Opiates are known to modulate the immune response directly and may increase susceptibility to infection ${ }^{[13]}$.

The specific types of drugs abused may differ in their impact on nutritional status, including weight. Among Hispanic drug abusers in Boston, men who used cocaine or cocaine and heroin, but not strict heroin or methadone users had a lower BMI compared to HIVpositive non-drug abusers ${ }^{[12]}$. The effect of cocaine was independent of infection with HIV or hepatitis. Cocaine has been associated with anorexia and eating disorders $^{[14]}$ and may have effects on energy intake, energy requirements or immunity that differ from the effect of opiates.

The commonly observed lower weight of drug abusers may or may not have deleterious consequences for health, depending on the whether weight falls within healthy limits. The weight of drug abusers appears to be increasing in parallel with the prevalence of overweight (BMI $\geq 25 \mathrm{~kg} \mathrm{~m}^{-2}$, ${ }^{[15]}$ ) in developed countries. Using autopsy data, the prevalence of overweight among Swedish drug abusers increased from $27.4 \%$ in 1988 to $45.5 \%$ in $2000^{[16]}$. In our study ${ }^{[11]}$, Hispanic HIVpositive and negative drug abusing men were overweight on average, while women were overweight to obese $\left(\mathrm{BMI} \geq 30 \mathrm{~kg} \mathrm{~m}^{-2}\right.$, $\left.{ }^{[15]}\right)$. Conversely, greater than $60 \%$ of drug abusers in Dhaka, Bangladesh had BMI below normal $\left(<18.5 \mathrm{~kg} \mathrm{~m}^{-2}\right)$ compared to normal controls $(15 \%)^{[9]}$. Thus, for those persons with advanced HIV or other wasting diseases, drug abuse may lead to severe weight loss or difficulties in weight recovery following bouts of illness. Even in the era of highly active antiretroviral therapy, weight loss is common $^{[17]}$ and low BMI continues to be a strong, independent predictor of mortality from $\mathrm{HIV}^{[18]}$.

Dietary intake of drug abusers: Studies of dietary intake have not provided clear evidence that energy deficits can explain the lower weight of drug abusers. Prior to the advent of HIV/AIDS, Gambera et al. ${ }^{[19]}$ examined the dietary intake of drug abusers in detoxification or methadone maintenance programs in San Francisco and found significant dietary deficiencies as compared to the recommended dietary allowance. Since that study did not have a control group, it is not clear whether the average intake of the drug abusers differed from that of drug abusers who were not enrolled in programs or non-abusers of similar lifestyle. Studies showing weight gain among drug abusers in detoxification programs cannot isolate the effect of program-induced changes in lifestyle, including diet, from changes in drug use ${ }^{[20]}$.

Other studies have looked at the diet of community-living drug abusers ${ }^{[3,7,10,11]}$. Morabia et al. ${ }^{[3]}$ used dietary histories to examine the food intakes of non-institutionalized heroin addicts. No differences in energy intake between heroin abusers and controls were found. Using the 24-hour dietary recall method, Smit et $a l .{ }^{[7]}$ found that energy intake was greater than estimated need in both HIV-positive and HIV-negative drug abusers, despite self-reported involuntary weight loss in both groups. Fourteen percent of participants reported consumption of one meal or less per day, while $86 \%$ reported at least two meals per day. These results agree with results from two studies conducted by our group $^{[10,11]}$. In our study of HIV-positive IVDU women $^{[10]}$, lower BMI was not explained by differences in dietary intake as measured by three-day food records: IVDU women reported average weight-adjusted energy intakes more that $53 \%$ higher than non-IVDU women. In a later study of Hispanics also by our group, the lower BMI of drug abusers could not be attributed to differences in average dietary energy intake as measured by three-day food records ${ }^{[11]}$. In a study of homeless Germans, drug abuse and wasting diseases, but not food intake, were associated poor nutritional status $^{[21]}$.

While drug abuse appears not to affect average energy intake, it may affect the frequency of meals and nutritional quality of the foods eaten ${ }^{[3,5,7,8,11,22,23]}$. Castro et $a l .{ }^{[22]}$ found that young adults who used cocaine ate fewer complete or balanced meals per day compared to young adults who did not use drugs. Our group found a non-statistically significant trend toward greater variability in average daily energy intakes with increasing frequency of drug use. This was reflected as more reported days of extremely low energy intake as well as more days of extremely high energy intake ${ }^{[11]}$.

Several studies have shown that, excluding calories from alcohol, drug abusers consume carbohydrates, including sweets, as a greater proportion of total energy intake $^{[3,8,11,22]}$. This may be due to food preferences. Weiss $^{[23]}$ found that incarcerated drug abusers fantasized about consumption of sweets and craved sweets more than other food types during periods of abstinence or prior to using drugs. In our study of Hispanic drug abusers ${ }^{[11]}$, HIV-negative drug abusing men, but not women, reported proportionally more carbohydrate and less protein in their diets compared to HIV positive non-drug abusers. However, differences in dietary carbohydrate were attributable to homelessness, not drug abuse per se. In the study of Hispanic women done by Himmelgreen et al. ${ }^{[8]}$, drug abusers were more likely to report food insecurity. Therefore, it appears that preference and homelessness or food insecurity may contribute to the relatively high carbohydrate intake of drug abusers. Emergency food banks and homeless shelters may provide meals that are high in carbohydrates.

Because dietary energy insufficiency did not seem to explain weight differences between drug abusers and non-drug abusers, we examined other, non-dietary factors that could affect the weight of drug abusers with and without $\mathrm{HIV}^{[12]}$. These factors included infection 
with hepatitis, malabsorption, increased resting energy expenditure and increased physical activity. We measured malabsorption using the two-hour D-xylose test and resting energy expenditure by indirect calorimetry. We assessed physical activity by asking the participants how many blocks they walked in a typical day. We also asked about weight lifting or body building exercises. While BMI was lower in men who used cocaine or cocaine and heroin together compared to men who did not use drugs, neither dietary energy intake nor any of the other non-dietary factors examined could explain the BMI differences among the men. A short-coming of our study was that we did not have complete measures of physical activity. Cocaine is known to increase fidgeting and restlessness and repetitive activity (termed "stereotypy") in rodents and humans ${ }^{[24,25]}$. It is possible that such small, but frequent amounts of physical activity could increase the energy requirements of cocaine users. Fidgeting has been shown to increase the energy requirements of normal, healthy individuals ${ }^{[26]}$ and could explain the observed lower BMI of cocaine users.

In summary, nutritional deficits, including weight deficits are reported among drug abusers in many studies, though the direct causes are still not clear. This implies that drug abusers with HIV and other wasting diseases may have difficulty maintaining their weight or regaining weight following bouts of illness. Since HIV-related weight loss is common, even in areas of the world where HAART is widely available, and weight loss is a strong predictor of mortality, further studies to elucidate underlying mechanisms are warranted.

\section{ACKNOWLEDGEMENTS}

This study was supported by the National Institute on Drug Abuse grants number DA 11598 and DA14501, NIDDK grant number DK45734, Center for AIDS Research number P30A142853, Center for Drug Abuse and AIDS Research 1P30DA13868. The General Research Center of the New England Medical Center, Boston, is supported by the Division of Research Resources of the National Institutes of Health, grant number M01-RR00054.

\section{REFERENCES}

1. Aylett, P., 1978. Some aspects of nutritional state in 'hard' drug addicts. Br. J. Nutr., 73: 77-81.

2. Altes, J., C. Dolz, A. Obrador and Forteza-Rei, 1988. Prevalence of protein-energy malnutrition in heroin addicts hospitalized for detoxication. J. Clin. Nutr. Gastroenerol., 3: 55-58.
3. Morabia, A., J. Fabre and E. Chee et al., 1989. Diet and opiate addiction: a quantitative assessment of the diet of non-I nstitutionalized opiate addicts. Br. J. Addict., 84: 173-180.

4. Varela, P., A. Marcos and S. Ripoll et al., 1990. Nutritional status assessment of HIV-positive drug addicts. Eur. J. Clin. Nutr., 44: 415-418.

5. Santolaria-Fernandez, F.J., J.L. Gomez-Sirvent and C.K. Gonzalez Reimers et al., 1995. Nutritional assessment of drug addicts. Drug Alcohol Depend., 38: 11-8.

6. McCombie, L., L. Elliot and K. Fasrrow et al., 1995. Injecting drug use and body mass index. Addiction, 90: 1117-21.

7. Smit, E., N.M.H. Graham and A. Tang et al., 1996. Dietary intake of community-based HIV-1 seropositive and seronegative injecting drug users. Nutrition, 12: 496-501.

8. Himmelgreen, D.A., R. Perez-Escamilla and S. Segura-Millan et al., 1998. A comparison of the nutritional status and food security of drug-using and non-drug using Hispanic women in Hartford, Connecticut. Am. J. Phys. Anthropol., 107: 351361.

9. Nazrul Islam, S.K., K.J. Hossain and A. Ahmed et al., 2002. Nutritional status of drug addicts undergoing detoxification: prevalence of malnutrition and influence of illicit drugs and lifestyle. Br. J. Nutr., 88: 507-513.

10. Forrester, J.E., M.N. Woods and T.K. Knox et al., 2000. Body Composition and dietary intake in relation to drug abuse in a cohort of HIV-positive persons. JAIDS, 25: S43-S48.

11. Forrester, J.E., K.L. Tucker and S.L. Gorbach, 2004. Dietary intake and body mass index in HIVpositive and HIV-negative drug abusers of Hispanic ethnicity. Public Health Nutr., 7: 863870 .

12. Forrester, J.E., K.L. Tucker and S.L. Gorbach, 2005. The effect of drug abuse on body mass index in Hispanics with and without HIV infection. Public Health Nutr., 8: 61-68.

13. Carr, D.J.J., T.J. Rogers and R.J. Weber, 1996. The relevance of opioids and opioid receptors on immunocompetence and immune homeostasis. Proc. Society for Experimental Biology and Medicine, 213: 248-257.

14. Jonas, J.M. and M.S. Gold, 1986. Cocaine abuse and eating disorders. Lancet, 1: 390-391.

15. World Health Organization, 1997. Obesity: Preventing and managing the global epidemic. Report of a WHO Consultation presented at the World Health Organization. June 3-5, 1997. Publication WHO/NUT/NCD/98.1. World Health Organization, Geneva, Switzerland. 
16. Rajs, J., A. Petersson and I. Thiblin et al., 2004. Nutritional status of deceased illicit drug addicts in Stockholm, Sweden-a longitudinal medicolegal study. J. Forensic Sci., 49: 320-9.

17. Wanke, C., M. Silva and T.A. Knox et al., 2000. Weight loss and wasting remain common complications in individuals infected with HIV in the era of highly active antiretroviral therapy. Clin. Infect. Dis., 31: 804-805.

18. Tang, A.M., J.E. Forrester and D. Spiegelman et $a l .$, 2002. Weight loss and survival in HIV-positive patients in the era of highly active antiretroviral therapy. J. Acquir. Immune. Defic. Syndr., 31: 230-236.

19. Gambera, S., 1976. Comments on dietary intake of drug-dependent persons. J. Am. Diet. Assoc., 68: 155-157.

20. Varela, P., A. Marcos and S. Ripoll et al., 1997. Effects of human immunodeficiency virus infection and detoxification time on anthropometric measurements and dietary intake of male drug addicts. Am. J. Clin. Nutr., 66: 509S-514S.
21. Langn $\triangleq$ se, K. and M.J. M $\hbar l l e r, ~ 2001$. Nutrition and health in an urban homeless population in Germany. Public Health Nutr., 4: 805-811.

22. Castro, F.G., M.D. Newcomb and K. Cadish, 1987. Lifestyle differences between young adult cocaine users and their nonuser peers. J. Drug Edu., 17: 8911 .

23. Weiss, G., 1982. Food fantasies of incarcerated drug users. The Intl. J. Addict., 17: 905-912.

24. Schiorring, E., 1981. Pathophysiology induced by 'speed drugs'. Pharmacol. Biochem. Behavior, 14 (Suppl. 1): 109-22.

25. Rosse, R.B., M. Fay-McCarthy and J.P.Jr. Collins et al., 1993. Transient compulsive foraging behavior associated with crack cocaine use. Am. J. Psychiat., 150: 155-156.

26. Levine, J.A., S.J. Schleusner and M.D. Jensen, 2000. Energy expenditure of non-exercise activity. Am. J. Clin. Nutr., 72: 1451-1452. 